\title{
ON THE FEASIBILITY OF A TRIPLER UPGRADE FOR LHC*
}

\author{
P. McIntyre ${ }^{\#}$ and A. Sattarov, Texas A\&M University, College Station, TX 77883 U.S.A.
}

\section{Abstract}

The design of high-field dipoles has been optimized using a block coil geometry. The optimization includes stress management and flux plate suppression of multipoles from snap-back. The design has been extended to higher field by devising a hybrid coil geometry containing inner windings of Bi-2212 and outer windings of $\mathrm{Nb}_{3} \mathrm{Sn}$. A 24 Tesla dual dipole using this design offers the possibility of an LHC tripler. Issues of fabrication technology and synchrotron radiation control are discussed. There is no obvious upper limit to the field that could be attained for the dipoles of future hadron colliders.

\section{THE HYBRID DIPOLE}

The LHC dipole reaches the highest performance that is possible with the classic technology of NbTi superconductor and $\cos \theta$ coil geometry. That methodology was first used in the Fermilab Tevatron, later in HERA and in RHIC, and now ultimately in LHC. Tripling the LHC field strength requires a new superconducting material and a new coil geometry.

The critical current density $j_{c}$ in a BCS superconductor decreases with field according to the Kramer relation, reaching about $20 \%$ of its low-field value at a field of $\sim 60 \%$ of $\mathrm{H}_{\mathrm{c} 2}$. In practical terms that is about the limit for using a superconductor in magnet coils: $\sim 8 \mathrm{~T}$ for $\mathrm{NbTi}$, and $\sim 16 \mathrm{~T}$ for $\mathrm{Nb}_{3} \mathrm{Sn}$. For winding fields beyond $16 \mathrm{~T}$ the high-temperature superconductor Bi-2212 has now been matured to a high-performance multi-filament round wire. Its $j_{c}$ is substantially field-independent to at least 35 Tesla.

This suggests a hybrid dipole strategy for extending dipole design to ever-higher field strength: use Bi-2212 cable for the inner windings where $\mathrm{B}>16 \mathrm{~T}$, and $\mathrm{Nb}_{3} \mathrm{Sn}$ for the outer windings where $\mathrm{B}<16 \mathrm{~T}$. The strategy is embodied in the $24 \mathrm{~T}$ dual dipole shown in Figure 1.

\section{STRESS MANAGEMENT}

To be successful such a strategy must also cope with the strain sensitivity of both materials. As field increases, Lorentz stress increases $\propto \mathrm{B}^{2}$. But $\mathrm{Nb}_{3} \mathrm{Sn}$ undergoes reversible degradation for stress $\sigma>150 \mathrm{MPa}$, and $\mathrm{Bi}-2212$ undergoes irreversible degradation for strain $\varepsilon>0.6 \%$.

Lorentz stress accumulates through the thickness of a superconducting coil. The field acts upon each cable element in turn and the forces add up as they are passed to the outside structure. This accumulation is unavoidable in coils of $\cos \theta$ geometry because the entire coil is one mechanical assembly.

A new generation of $\mathrm{Nb}_{3} \mathrm{Sn}$ dipoles has been under de-

\footnotetext{
*Work supported by US Dept. of Energy, grant \# DE-FG03-95ER40924, and by EC Research Infrastructure Activity FP6: 'Structuring. the European Research Area' program, CARE-RII3-CT-3003-506395.

"p-mcintyre@physics.tamu.edu
}

velopment, in which the coils are arranged in rectangular racetrack windings and a support matrix of ribs and plates made of the high-strength alloy Inconel 718 is integrated within the windings [1].

The support matrix intercepts the forces acting on inner windings and bypass them past outer windings to the flux return. The strategy is summarized in Figure 2. Three windings in a horizontal section are shown, with ribs and plates of high-strength Inconel providing the support matrix. A preload is applied to the structure from the left, and Lorentz forces push from the right. A laminar spring is located at the inner end of each winding to enforce the decoupling of stress from one winding to the next. The laminar spring is made of tempered Inconel X-750, which retains a spring temper through a sustained $850 \mathrm{C}$ bake.

Shear is released by lining all windings with mica paper. Thus even when the overall Lorentz stress exceeds $300 \mathrm{MPa}$, the stress in the windings never exceeds $\sim 150$ $\mathrm{MPa}$.

Stress management is also provided for the preload of the coil assembly using a pattern of expansion bladders. Thin stainless steel bladders are inserted along the four flat interface between the coil assembly and the flux return. A pair of curved bladders is located between the flux return and the outer aluminum stress tube. After final assembly of the dipole, the entire dipole is heated to $\sim 90$ $\mathrm{C}$ and the bladders are evacuated and then filled with molten Wood's metal. The molten metal is pumped to a pressure corresponding to the desired preload and then the magnet is cooled while maintaining hydraulic pressure on the bladders. The Wood's metal alloy is selected to have net zero expansion over the cycle from melt temperature to $4 \mathrm{~K}$, so preload is preserved in the operating dipole. This has the remarkable result that a totally uniform preload is delivered throughout the interfaces.

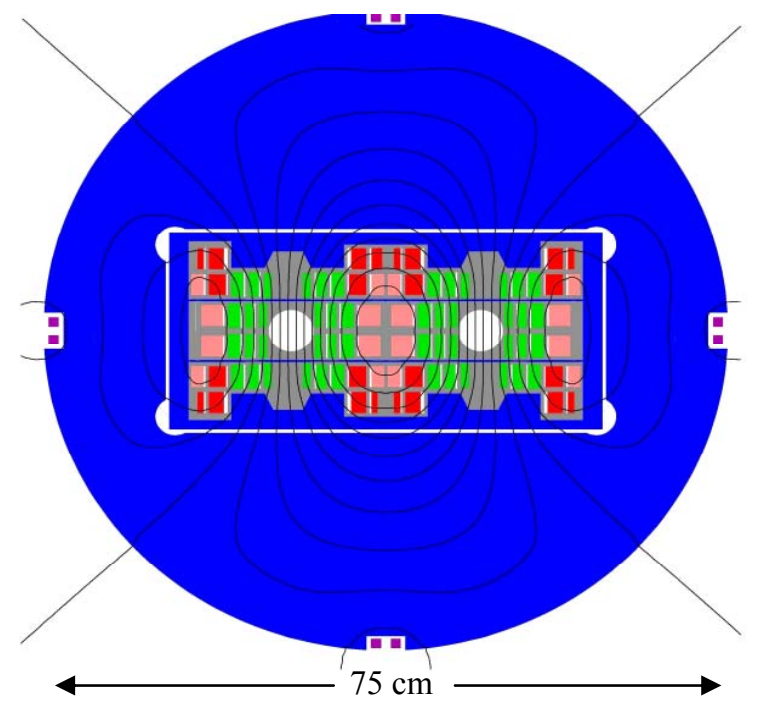

Figure 1. 24 Tesla hybrid dipole for LHC tripler. 

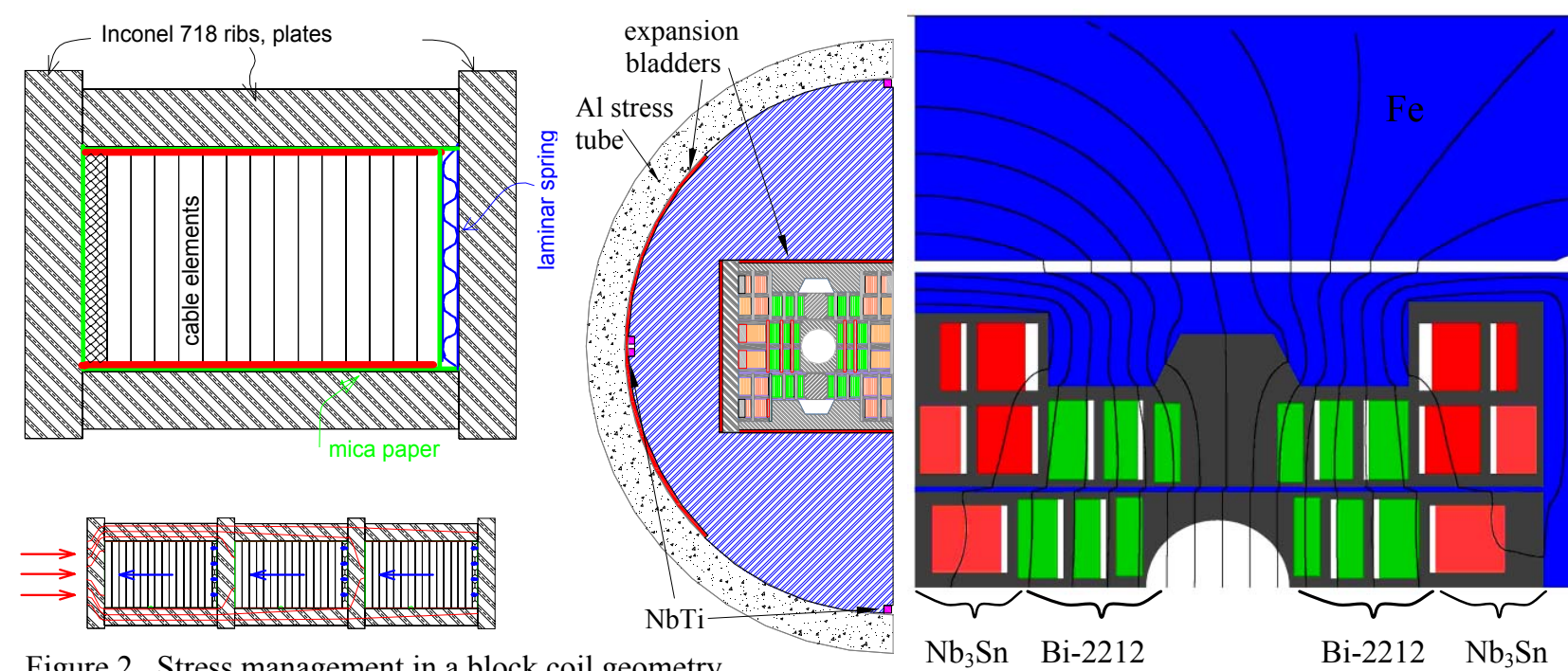

Figure 2. Stress management in a block coil geometry.

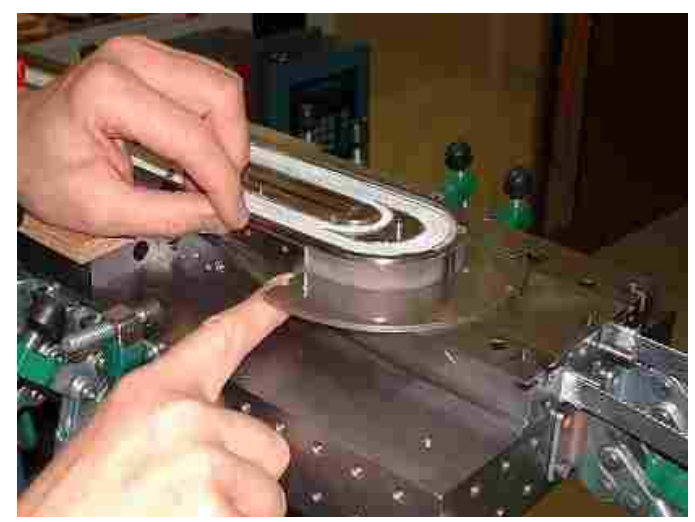

Figure 4. Elements of the hybrid coil dipole: a) halfsection showing bladders; detail showing flux plate.

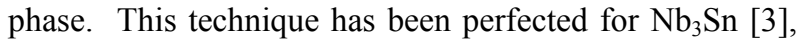
for which the necessary heat treat is at $\sim 650 \mathrm{C}$ in an argon atmosphere. The optimum heat treatment for $\mathrm{Bi}-2212$ is very different, however [4]. The winding must be heated to $\sim 850 \mathrm{C}$ in an oxygen-rich atmosphere (to push the stoichiometry of the superconducting phase), and the heat treatment culminates in a brief excursion (only $\sim 5$ minutes!) into partial melt of the 2212 phase at $\sim 870$ C. Such temperature control would be problematic in large coils.

Instead the partial melt heat treat of the Bi-2212 windFigure 3. Applying laminar springs, ribs and plates to inner winding of $\mathrm{Nb}_{3} \mathrm{Sn}$.

Figure 3 shows the actual fabrication of a winding embodying stress management. The innermost winding is complete, and a laminar spring is being installed on a support rib preparatory to winding the second winding.

Figure $4 \mathrm{a}$ shows a detail of the winding assembly for the $24 \mathrm{~T}$ hybrid dual dipole. Figure $4 \mathrm{~b}$ illustrates the use of a flux plate to suppress multipoles from persistent currents and snap-back [2] at injection field. The flux plate is a horizontal steel sheet located between the upper and lower rows of windings. At injection field (1.25 T corresponds to $1 \mathrm{TeV}$ for an LHC tripler) this sheet is unsaturated and imposes a dipole boundary condition that suppresses all higher multipoles in the beam tube region. Simulations of this feature indicate a reduction of sextupole by a factor of 5 . The suppression offsets the increase in magnetization that would result from the larger subelements in $\mathrm{Nb}_{3} \mathrm{Sn}$ and $\mathrm{Bi}-2212$ strands compared to $\mathrm{NbTi}$.

A set of NbTi windings are located in notches in the outside surface of the steel as shown in Figure 4b. These windings are used to contain return flux within the steel.

\section{HEAT TREATMENT OF $\mathrm{Nb}_{3} \mathrm{Sn}, \mathrm{Bi}-2212$}

Both $\mathrm{Nb}_{3} \mathrm{Sn}$ and $\mathrm{Bi}-2212$ windings must be heat treated in a wind-and-react process to form the superconducting ings will be done using the isothermal melt process invented by Holesinger [4]. Once the winding has been preheated to just below the melt transition of the 2212 phase, the transition into partial melt can be induced by increasing the partial pressure of oxygen $\left(\mathrm{pO}_{2}\right)$ in the purge gas instead of by changing the temperature. After the desired time in partial melt, $\mathrm{pO}_{2}$ is returned to its previous value, restoring the solid phase. By providing parallel flow of purge gas throughout the winding assembly, the partial melt transition can be controlled to follow the same time progression throughout the entire winding assembly.

The challenge for a hybrid coil is that one must accomplish both of these heat treats in the same hybrid coil. At high temperature oxygen would ruin the stabilizing copper sheath on $\mathrm{Nb}_{3} \mathrm{Sn}$, but a lack of oxygen would deplete the stoichiometry of the Bi-2212 and ruin its superconducting performance.

Table 1. Main parameters of the $24 \mathrm{~T}$ hybrid dipole.

Dipole dimensions:

$$
\begin{aligned}
& \text { length } \\
& \text { cold mass diameter }
\end{aligned}
$$

Beam tube diameter

Operating temperature

Coil current

Maximum stress in windings

Stored energy/bore

Total horizontal Lorentz force/bore
$30 \mathrm{~m}$

$80 \mathrm{~cm}$

$40 \mathrm{~mm}$

$4.5 \mathrm{~K}$

$33 \mathrm{KA}$

$150 \mathrm{MPa}$

$5 \mathrm{MJ} / \mathrm{m}$

$40 \mathrm{MN} / \mathrm{m}$ 
We have devised a procedure that resolves this difficulty. All of the Bi-2212 windings will be completed and supported within their stress management structure, and the Bi-2212 heat treatment will be performed using the isothermal melt process. The $\mathrm{Nb}_{3} \mathrm{Sn}$ windings will then be wound onto the completed Bi-2212 structure. The reaction bake for the $\mathrm{Nb}_{3} \mathrm{Sn}$ windings will be performed while maintaining an oxygen-rich purge on the Bi-2212 windings and an Ar purge on the $\mathrm{Nb}_{3} \mathrm{Sn}$ windings. The box-like support matrix that is provided for stress management has the useful double-purpose of providing internal isolation within the coil assembly between the two winding regions. Purge gas flow can be channeled separately through the two coil regions - oxygen through the inner Bi-2212 windings and argon through the outer Bi2212 windings.

Perhaps the most obvious challenge for an LHC Tripler is the strong dependence of synchrotron radiation upon beam energy. The power per unit length $\widetilde{P}$ radiated by a proton beam of energy $E$, and curvature radius $\rho$ is $\widetilde{P} \propto E^{4} / \rho^{2}$. Tripling the energy while keeping $\rho$ constant increases $\widetilde{P}$ from $0.22 \mathrm{~W} / \mathrm{m}$ to $14 \mathrm{~W} / \mathrm{m}$. Already in LHC absorbing the synchrotron radiation is a major challenge, requiring an intermediate-temperature beam screen within the beam tube. It would seem at first that this huge increase in $\widetilde{P}$ would kill a Tripler.

But the spectrum of synchrotron light also hardens. The critical energy $E_{c}$ is the peak of this spectrum: $E_{c} \propto E^{3} / \rho$. Tripling the energy increases $\mathrm{E}_{\mathrm{c}}$ from 44 $\mathrm{eV}$ (hard UV) to $1.2 \mathrm{keV}$ (soft X-ray). The hard UV photons in LHC have significant cross-section for backscattering from the surface layers of the beam tube and photoionization [5]. By contrast soft X-rays in the Tripler penetrate into the surfaces and stop within the first $\sim 100 \mu \mathrm{m}$.

A photon stop is proposed to absorb the synchrotron light fan from each dipole at several discrete locations along its length. The design is shown in Figure 5a. A total of 3 photon stops are provided for each $30 \mathrm{~m}$ dipole: two along the dipole length and the third at its end. A beam screen is installed along the length of the dipole to intercept specular reflections at a temperature of $\sim 20 \mathrm{~K}$.

Each photon stop within the dipole is mounted in a $\mathrm{T}$ insertion to the beam tube, supported within a $2.5 \mathrm{~cm}$. diameter tube extending up through the entire structure of the dipole. The photon stop is maintained at $160 \mathrm{~K}$ by refrigeration with liquid xenon (LXe). The photon stop consists of a blade supported on a stem which contains the supply and return LXe flows. The stem is mounted on a rotary feedthrough and can be rotated between two angular positions (Figure 5a): in the out-position it provides maximum clearance $(\sim 1.8 \mathrm{~cm})$ for injection of beams (Figure 5b); in the in-position it intercepts synchrotron light to within a few $\mathrm{mm}$ of the beam (Figure 5c). Preliminary calculations by F. Zimmermann indicate that, if a return foil is attached to the downstream edge of each photon stop to carry image current back to the beam screen, the effect on impedance is modest.

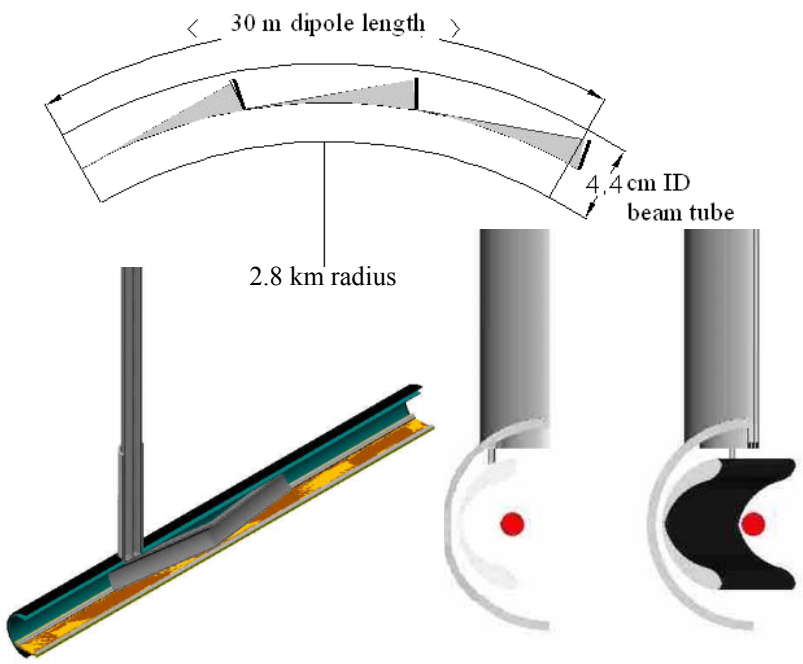

Figure 5. Photon stop in dipole: a) placement along dipole; b) cutaway view; end view at c) iniection; d) collision.

Each photon stop must intercept a photon power $P_{\gamma}=\widetilde{P} L=100 \mathrm{~W}$. The blade shown in Figure $5 \mathrm{~b}$ has a length of $20 \mathrm{~cm}$ and LXe cooling lines in of the top and bottom lips. The heat transfer corresponds to a surface density of $\sim 1 \mathrm{~W} / \mathrm{cm}^{2}$. The radiant heat load to the beam screen from each photon stop is $\sim 0.1 \mathrm{~W}$. The a.c. power to remove the heat of Tripler's synchrotron light at $160 \mathrm{~K}$ is comparable to the installed capacity for LHC.

\section{CONCLUSIONS}

A conceptual design for a $24 \mathrm{~T}$ hybrid dipole has been prepared. While much work will be required to develop the processes for heat treatment and splices, the design builds upon the techniques of stress management and magnetics that have been developed for $\mathrm{Nb}_{3} \mathrm{Sn}$ dipoles. The hybrid dipole would make possible a Tripler upgrade to LHC. Studies of phenomenology of a Tripler upgrade suggests that it would double the mass reach for discovery of new gauge fields. With the proposed approach to stress management and synchrotron light; there appears to be no foreseeable limit to how high a magnetic field could be useful for future hadron colliders.

\section{REFERENCES}

[1] N. Diaczenko et al., 'Stress management in highfield dipoles', Proc. PAC1997.

[2] S. Sanfilippo, 'Persistent and coupling current effects in the LHC superconducting dipoles', Proc. Appl. Superconductivity Conf., Houston, TX, August 4-9, 2002.

[3] R.A. Scanlan, 'Superconducting Materials for the Next Generation Colliders', VLHC Magnet Technologies Workshop, Fermilab, May 24-26, 2000.

[4] T.G. Holesinger et al., 'Microstructural development in Bi-2212 tapes by conventional and isothermal melt processing', Proc. Appl. Superconductivity Conf., 2000.

[5] R. Cimino, I.R. Collins, and V. Baglin, Phys. Rev. Special Topics - Accelerators \& Beams 2, 063201 (1999). 